\title{
Smart Nanotextiles: Materials and Their Application
}

\section{Introduction}

Textiles are ubiquitous to us, enveloping our skin and surroundings. Not only do they provide a protective shield or act as a comforting cocoon but they also serve aesthetic appeal and cultural importance. Recent technologies have allowed the traditional functionality of textiles to be extended. Advances in material science have added intelligence to textiles and created "smart" clothes. Smart textiles can sense and react to environmental conditions or stimuli, e.g. from mechanical, thermal, chemical, electrical or magnetic sources. Such textiles find uses in many applications ranging from military and security to personalised healthcare, hygiene and entertainment.

Smart textiles may be termed "passive" or "active". A passive smart textile monitors the wearer's physiology or the environment e.g. a shirt with in-built thermistors to log body temperature over time. If actuators are integrated the textile becomes an active smart textile as it may respond to a particular stimulus, e.g. the temperature aware shirt may automatically rolls up the sleeves when body temperature becomes elevated.

The fundamental components in any smart textile are sensors and actuators. Interconnections, power supply and a control unit are also needed to complete the system. These components must all be integrated into textiles while still retaining the usual tactile, flexible and comfortable properties that we expect from a textile.

Adding new functionalities to textiles while maintaining the look and feel of the fabric is where nanotechnology is having a huge impact on the textile industry. This article describes current developments in materials for smart nanotextiles and some of the many applications where these innovative textiles are of great benefit.

\section{Materials}

Materials such as metallics, optical fibres and conductive polymers may be integrated into the textile structure, thus supplying electrical conductivity, sensing capabilities and data transmission. Development of electronic textiles must take into account traditional textile manufacturing techniques in addition to the properties of the finished garment. Organic polymers may provide a solution to overcome the stiffness of inorganic crystals such as silicon. These materials are light, elastic, resilient, mechanically flexible, inexpensive and easy to process.

\section{II.A Metal fibers}

Metal threads are made up of metal fibres which are very thin metal filaments (diameters ranging from 1 to 80 micron). The fibers are produced either through a bundle-drawing process or else shaved off the edge of thin metal sheeting. Metallic threads and yarns may be knitted or woven into a textile and used to form interconnects between components. They may also be used as electrodes for monitoring electrical physiological activity such as electrocardiogram (ECG) signals. While metals provide good conductivity there are some drawbacks of integration into clothing. Metal threads tend to be heavier than most textile fibers and their brittle characteristics can damage spinning machinery over time and also they may be uncomfortable to wear due to abrasion.

\section{II.B Conductive inks}


A layout can be screen-printed using conductive inks to add conductivity to specific areas of a garment. Carbon, copper, silver, nickel and gold may be added to conventional printing inks to make them conductive. Printed areas can be subsequently used as switches or pressure pads for the activation of circuits.

\section{II.C Quantum tunnelling composites}

Quantum Tunnelling Composites (QTCs) have the capability of transforming from a virtually perfect insulator to metal like conductor when deformed. QTCs are manufactured by Peratech Ltd. The fabrics consist of a layered structure. Unperturbed, the conductive layers are too far apart to conduct electricity but when pressure is applied, they move closer and electrons can tunnel through the insulator. The resistance of QTCs can drop gradually over an unprecedented range from greater than $10^{12}$ ohms to less than one ohm. Softswitch ${ }^{\mathrm{TM}}$ and Eleksen ${ }^{\circledR}$ have made use of this material as a textile switch in a number of products including jackets with iPod control and fabric keyboards.

\section{$<$ Figure 1>}

\section{II.D Inherently conductive polymers}

Inherently conductive polymers have both sensing and actuation properties. The electrical conductivity of these polymers is known to be caused by their conjugated double bond chain structure. Some commonly known ICPs include polyacetylene, polypyrrole, polyaniline, and polythiophene. Polypyrrole (PPy) is most suitable as it has high mechanical strength with high elasticity, is relatively stable in air and electroactive in both organic and aqueous solutions. Polypyrrole has be used in the development of organic piezoresistive sensors by depositing thin layers of PPy, by an in situ chemical polymerization process, onto fabrics with high elasticity such as nylon lycra ${ }^{\circledR}$ or high compressibility such as polyurethane foam. Conductivity changes results from external deformation of the material. The major advantage of this approach is that the sensors retain the natural texture of the material. However the problem with these devices is a variation in resistance over time, and a high response time. Another approach to developing piezo-resistive polymer sensors demonstrated by the University of Pisa using carbon loaded elastomers. A conductive mixture of silicone and carbon black powder has been used to develop a number of sensorized garments for high performance sensing with a reported GF of 2.5, similar to metals, and TCR value of $0.08^{\circ} \mathrm{C}^{-1}$.

In order to create fabrics with motor functions flexible actuating devices are needed. ICPs have been given the name "Artificial Muscles" as they have the ability to emulate biological muscles. Although the current actuation force and mechanical energy density of ICPs are relatively low, there is potential to develop rehabilitation and orthotic devices.

\section{II.E Optical fibers}

Plastic optical fibres may be easily integrated into a textile. They have the advantage of not generating heat and are insensitive to EM radiation. Optical fibres may serve a number of functions in a smart garment - transmit data signals, transmit light for optical sensing, detect deformations in fabrics due to stress and strain and perform chemical sensing. Plastic optical fibres can be woven into a textile, however bending of the fibers is an issue during the manufacturing process and also with the end 
product as mechanical damage causes signal loss. Commercially available Luminex ${ }^{\circledR}$ fabric is a textile with woven optical fibers capable of emitting its own light. While this has aesthetic appeal for the fashion industry it is also used in safety vests and has the potential to be used for data transmission.

\section{II.F Coating with nano-particles}

Coating a fabric with nanoparticles is being widely applied within the textile industry to improve the performance and functionality of textiles. Conventional methods of adding various properties to fabrics may not last after washing and wearing. However nanotechnology can add permanent effects and provide high durability fabrics. This is due to the large surface area-to-volume ratio and high surface energy of nanoparticles. Coating with nano-particles can enhance the textiles with properties such as anti-bacterial, water-repellence, UV-protection and self-cleaning, while still maintaining breath-ability and tactile properties of the textile. Nano-Tex has a range of products using such coatings to resist spills, repel and release stains, and resist static. These textile enhancements become inherent to the fabric, improving the performance and durability of everyday apparel and interior furnishings.

\section{II.G Organic Semiconductors}

Organic semiconductors, (polymers and oligomers), having the electrical properties of semiconductors and the mechanical properties of plastics, are good candidates for developing electronic and optoelectronic flexible components, e.g. transistors, LEDs, on the flexible textile substrate. Organic LEDs consist of multilayer structures where organic emitters are embedded between an evaporated metal electrode and a film of indium tin oxide coupled to a plastic or glass substrate. Philips have recently released a light emitting fabric, Lumalive ${ }^{\circledR}$, featuring flexible arrays of fully integrated coloured LEDs. These light-emitting textiles can carry dynamic messages, graphics, or multicoloured surfaces.

\section{II.H Shape Memory Fabrics}

Shape memory fabrics are materials that are able to return to a pre-programmed shape with the right stimuli, normally temperature. Two approaches may be taken to develop shape memory materials - using shape memory alloys (SMAs) or shape memory polymers (SMPs). In the case of shape memory alloys at low temperature the structure of the materials is in a martensite phase that can be easily deformed. Upon heating, the structure changes to an austenite phase, and the programmed shape is recovered as the material "remembers" its original shape. SMAs may be spun in combination with traditional fibres to create bi-component yarns which can then be woven or knitted. SMPs on the other hand have higher extensibility, superior processability, lower weight and a softer handle, and therefore may be considered more suitable for the clothing industry. Coating a fabric with SMP creates "non-iron" garments that can return to the original shape when heated.

\section{Applications}

\section{III.A Sports and Human Performance}

The sports sector, through seeking to improve athletic performance, personal comfort and protection from the elements, has driven significant research activity within the textile industry, e.g. breathable waterproof fabrics such as Goretex ${ }^{\circledR}$ and moisture management textiles like Coolmax ${ }^{\circledR}$. It is even possible to maintain constant body 
temperature using phase-change technology used in Outlast Adaptive Comfort ${ }^{\circledR}$ where excess body heat is absorbed, stored and released when needed. Clothes are increasingly able to adapt dynamically to the needs of the wearer. The latest developments integrate sensing capabilities to provide instantaneous awareness of the physiological condition of the athlete, thus providing valuable information about the athlete's physical abilities, training status, athletic potential, and responses to various training regimens. Demand for wearable sensors that can be used in real situations for kinematic analysis, vital sign monitoring and biochemical analysis is expanding rapidly, as the technology becomes increasingly viable.

Strain sensors, made from piezo-electric materials may be used in biomechanical analysis to realize wearable kinesthetic interfaces able to detect posture, improve movement performance and reduce injuries. Such devices may be used to teach athletes the correct way to perform movement skills by providing real-time feedback about limb orientation.

A new and exciting area of research that will have major impact for sports performance involves integrating chemical sensors into textiles. The aim of the EUsupported BioTex project is to perform real-time analysis of various constituents in sweat. For effective rehydration strategies it is important not only to replace volume losses, but also electrolytes.

\section{III.B Personalised Healthcare}

The concept of personalised healthcare empowers the individual with the management and assessment of their own healthcare needs. Wearable devices allow physiological signals to be continuously monitored during normal daily activities. This can overcome the problem of infrequent clinical visits that can only provide a brief window into the physiological status of the patient. Smart clothing serves an important role in remote monitoring of chronically ill patients or those undergoing rehabilitation. It also promotes the concept of preventative healthcare. Given the current world demographics there is a need to shift the focus of healthcare delivery from treatment to prevention, and also to promote wellness monitoring rather than diagnosis of illness.

Two EU-funded projects, WEALTHY and MyHeart, involve a wearable textile interface integrating sensors, electrodes and connections realised with conductive and piezoelectric yarns for monitoring vital signs. New products coming onto the market for similar applications include the Smartshirt by Sensatex ${ }^{\mathrm{TM}}$ and the Life Shirt ${ }^{\circledR}$ system by Vivometrics ${ }^{\circledR}$, offering continuous ambulatory monitoring systems for the healthcare sector.

\section{$<$ Figure 2>}

Traditionally, textiles have been regarded as an essentially passive 'skin' that provides enhanced protection, comfort and appearance, whereas smart textiles have the potential to emulate and augment the skin's sensory system by sensing external stimuli such as proximity, touch, pressure, temperature and chemical/biological substances. For conditions such as diabetes mellitus, where the patient loses sensation in the limbs, or bedridden patients, pressure sensitive fabrics may aid in assessment and warning to reduce the occurrence of pressure ulcers. With nanotechnologies, 
smart textiles may provide a fully functioning haptic interface - potentially a second, more sensitive skin.

Novel functionalities in textiles are of course not limited to personal apparel. Home furnishings may be enlisted into ubiquitous sensing within smart homes for telemonitoring of elderly, convalescent or isolated communities. This is an integral component in the"continuity of care" concept that textile based sensing can provide through monitoring patients in their home environment, and familiar surroundings.

\section{III.C Military/security}

In extreme environmental conditions and hazardous situations there is a need for realtime information technology to increase the protection and survivability of the people working in those conditions. Improvements in performance and additional capabilities would be of immense assistance within professions such as the defense forces and emergency response services. The requirements for such situations are to monitor vital signs and ease injuries while also monitoring environment hazards such as toxic gases. Wireless communication to a central unit allows medics to conduct remote triage of casualties to help them respond more rapidly and safely.

\section{III.D Fashion/lifestyle}

The development of high-tech advanced textiles for initial high-value applications such as extreme sports will eventually find its way into street fashion, with designers employing their creativity to use these emerging materials in new ways. We are becoming increasingly reliant on technology carrying MP3 players, laptops, mobile phones and digital cameras. These devices all contain common components such as power supply, microprocessor, data transmission. As the technology is becoming more flexible these could ultimately be integrated into a common textile substrate our clothes, becoming truly portable devices. Already there are textile switches integrated into clothing for the control of such devices.

While technology may be hidden through invisible coatings and advanced fibers, it can also be used to dramatically change the appearance of the textile, giving new and dazzling effects. Light emitting textiles are finding their way onto the haute couture catwalks, suggesting a future trend in technical garments.

\section{$<$ Figure 3>}

\section{Conclusions}

The advent of wearable technologies will affect many aspects of our daily lives and by the development smart nanotextiles such technologies will allow innocuous sensing of the wearer and their environs. A major challenge in wearable computing at present is how to interconnect components and to find alternatives to conventional silicon and metal components which are difficult to integrate with soft textile substrates. Smart textiles must be flexible enough to be worn for long periods of time, without causing any discomfort to the wearer. This is critical in order to create viable and accessible products. The way forward is to integrate materials at the nanoscale level, as this preserves the the flexible characteristics and tactile properties that we expect from our clothing. 
Smart textiles will impact on a huge range of applications, often starting at a highly specialized application before becoming commonly available to the general consumer. In order for this to happen, research must be broadly interdisciplinary ranging across materials research, sensor technologies, engineering, wireless networking, computer applications. Furthermore, to create something truly wearable input is needed from textile and fashion designers, and manufacturers. The needs of each target application must be assessed in conjunction with the end users such as healthcare professionals, the defense forces and sports physicians. There are so many potential applications where smart nanotextiles may impact on our lifestyles and become ubiquitous in this technology driven world.

\section{Bibliography}

1. Axisa, F., P.M. Schmitt, C. Gehin, G. Delhomme, E. McAdams, and A. Dittmar, (2005), Flexible Technologies and Smart Clothing for Citizen Medicine, Home Healthcare and Disease Prevention. IEEE Transactions on Information Technology in Biomedicine. 9(3): p. 325-336.

2. Bonato, P., (2003) Wearable Sensors/Systems and Their Impact on Biomedical Engineering, in IEEE Engineering in Medicine and Biology Magazine.p. 1820.

3. Braddock Clarke, S.E. and M. O'Mahony, (2005) Techno Textiles 2. Thames \& Hudson Ltd.

4. Brady, S., L. Dunne, A. Lynch, B. Smyth, and D. Diamond, Wearable Sensors? What is there to sense? (2006) Stud Health Technol Inform, 117: p. 80-88.

5. Carpi, F. and D. De Rossi, 2005, Electroactive Polymer-Based Devices for eTextiles in Biomedicine. IEEE Transactions on Information Technology in Biomedicine. 9(3): p. 295-318.

6. Engin, M., A. Demirel, E.Z. Engin, and M. Fedakar, 2005, Recent developments and trends in biomedical sensors. Measurement. 37: p. 173-188.

7. Lam Po Tang, S. and G.K. Stylios, (2006), An overview of smart technologies for clothing design and engineering. International Journal of Clothing Science and Technology. 18(2): p. 108-128.

8. Post, E.R., M. Orth, P.R. Russo, and N. Gershenfield, (2000) E-broidery: Design and fabrication of textile-based computing. IBM Systems Journal, 39(3\&4): p. 840-859.

9. Qian, L. and J.P. Hinestroza, (2004), Application of Nanotechnology for high performance tectiles. Journal of Textile and Apparel, Technology and Management, 4(1): p. 1-7.

10. Tao, X.M., 2005, Wearable electronics and photonics, Woodhead Publishing.

11. Troester, G., 2005, The Agenda of Wearable Healthcare, in IMIA Yearbook of Medical Informatics, R. Haux and C. Kulikowski, Editors, Schattauer: Stuttgart. p. 125-138.

12. Wong, Y.W.H., C.W.M. Yuen, M.Y.S. Leung, S.K.A. Ku, and H.L.I. Lam, (2006), Selected Applications of Nanotechnology in Textiles. AUTEX Research Journal, 6(1): p. 1-8.

Figure 1

Fabric keyboard using quantum tunnelling composite textile, produced by Eleksen (Courtesy of Peratech) 
Figure 2

On-body sensors and electronics for monitoring vital body signs - a garment with textile ECG electrodes developed by the MyHeart project to prevent heart failure. (Source : Philips)

Figure 3

Optical fibres are woven to create a light-emitting textile, (Courtesy of Luminex)
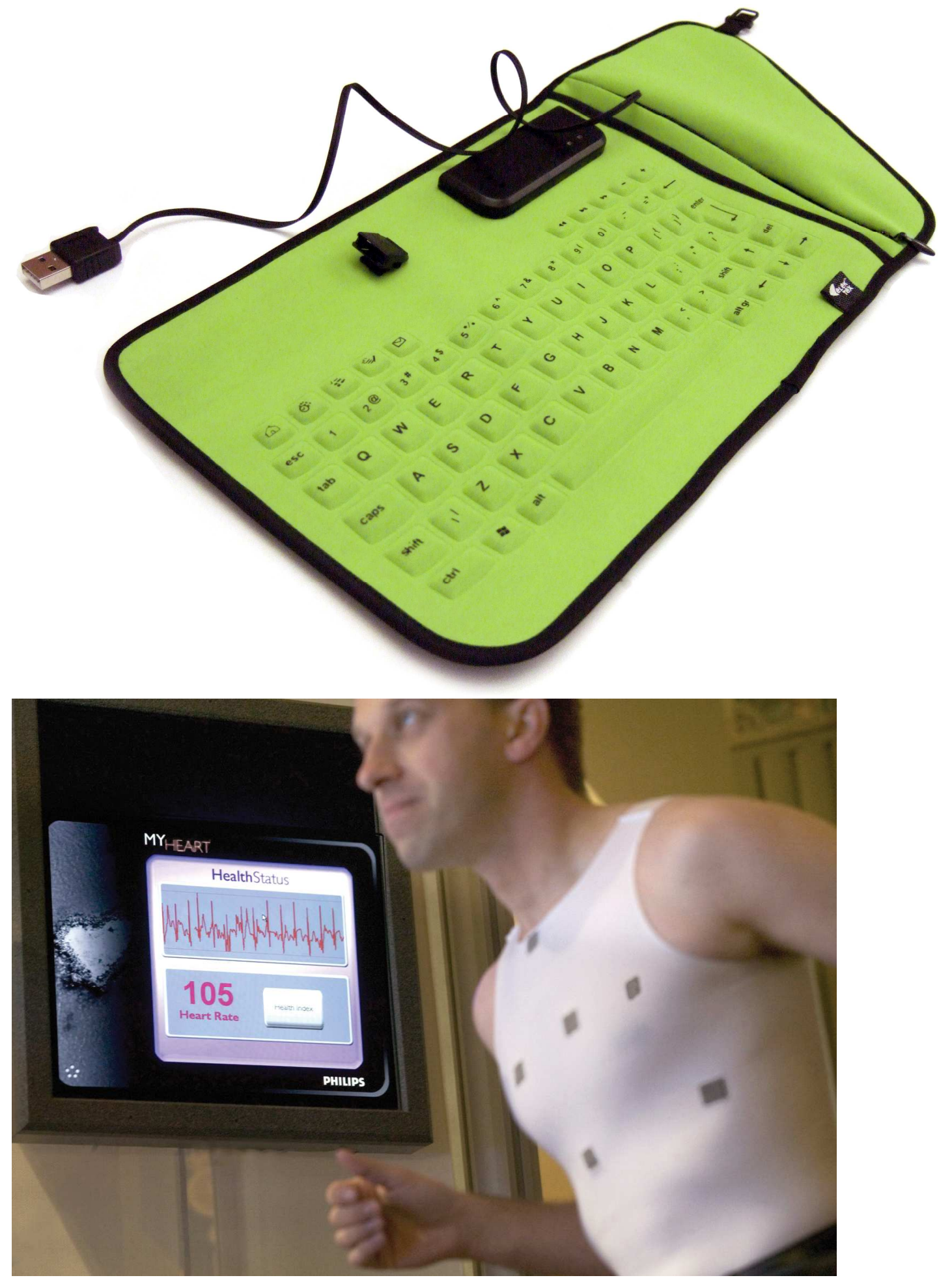


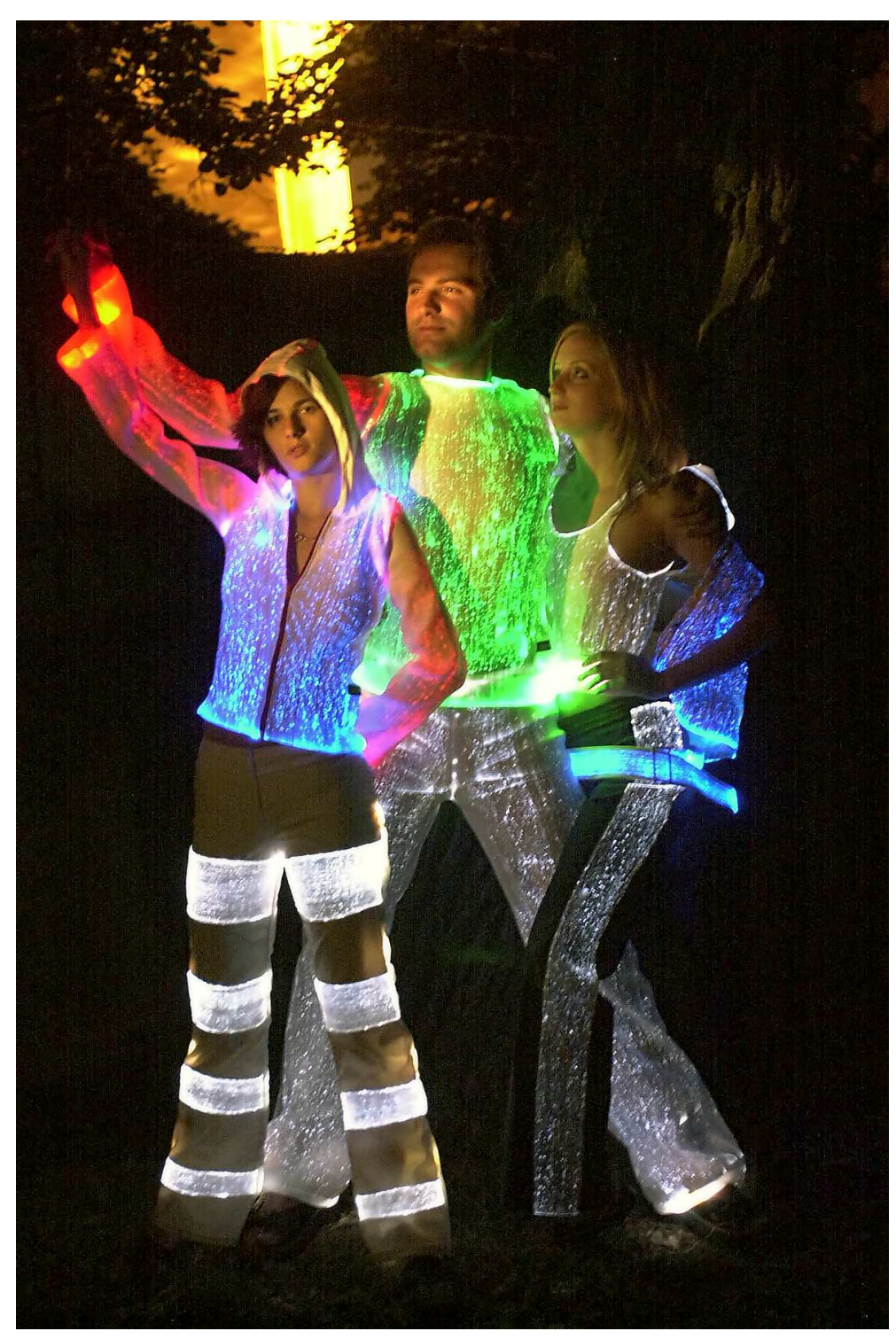

